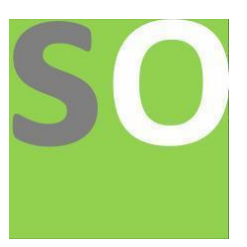

Article title: Extended Bandwidth and Increased Efficiency Quasi-Doherty Power Amplifier Design With A Revised Approach For Load Modulation

Authors: Seyedehmarzieh Rouhani[1], Kasra Rouhi[2], Adib Abrishamifar[3], Majid Tayarani[4]

Affiliations: Iran University of Science and Technology[1]

Orcid ids: 0000-0002-5362-5624[1], 0000-0002-1226-0104[2]

Contact e-mail: smrouhanie@elec.iust.ac.ir

License information: This work has been published open access under Creative Commons Attribution License http://creativecommons.org/licenses/by/4.0/, which permits unrestricted use, distribution, and reproduction in any medium, provided the original work is properly cited. Conditions, terms of use and publishing policy can be found at https://www.scienceopen.com/.

Preprint statement: This article is a preprint and has not been peer-reviewed, under consideration and submitted to ScienceOpen Preprints for open peer review.

DOI: 10.14293/S2199-1006.1.SOR-.PPBWXHI.v1

Preprint first posted online: 19 April 2021

Keywords: Doherty, High Efficiency, Load Modulation, PAE, Wideband 


\title{
Extended Bandwidth and Increased Efficiency Quasi-Doherty Power Amplifier Design With A Revised Approach For Load Modulation
}

\author{
Seyedehmarzieh Rouhani, Kasra Rouhi, Adib Abrishamifar ${ }^{1}$, Majid Tayarani \\ School of Electrical Engineering, Iran University of Science and Technology, Tehran, Iran, \\ Tel: +98(21) 73225727, Fax: +98(21) 73225777
}

\begin{abstract}
This paper presents an approach to power added efficiency (PAE) increase for Quasi-Doherty power amplifier (Q-DPA) design. For this aim, active feedback is utilized instead of a passive quarter wavelength transmission line $(\lambda / 4 \mathrm{TL})$ usage, which is conventionally used in the DPA schematic. PAE increase can be done by applying an accurate load modulation to the main amplifier ( $\left.\mathrm{PA}_{\text {main }}\right)$, especially for technologies in which output impedance of the main power amplifier $\left(Z_{\text {out,main }}\right)$ considerably varies in both low and high power regions. Because such precise modulation is still based on a modified TL, this approach suffers from the inherent narrowband behavior of that TL. As a consequence, expecting a wideband DPA may not be satisfied in all cases. To deal with this issue, active feedback is used to play a role in reaching $\mathrm{PA}_{\text {main, }}$ which is not saturated before, to its maximum efficiency at the highest level of received input power $\left(\mathrm{P}_{\text {in }}\right)$ in the high power region. Following $\mathrm{Z}_{\mathrm{out} \text {,main }}$ trajectories in power and frequency sweeps simultaneously just by a passive TL are not needed anymore. Still, for the sake of preventing total PAE degradation due to the consummated power by the feedback path's power amplifier ( $\left.\mathrm{PA}_{\text {feedback}}\right)$ should be limited, analytical confinement is provided in this work. A comparison is made between GaAs pHEMT 0.25um MMIC technology-based conventional DPA and the
\end{abstract}

\footnotetext{
${ }^{1}$ Corresponding author.

E-mail: smrouhanie@elec.iust.ac.ir (S.Rouhani), kasrarouhi@elec.iust.ac.ir (K.Rouhi), abrishamifar@iust.ac.ir (A.Abrishamifar), m_tayarani@iust.ac.ir (M.Tayarani)
} 
proposed revised approach based-DPA to verify the mentioned approach. The proposed PA shows maximum output power of $33.4 \mathrm{dBm}$, maximum PAE of 41.6, fractional bandwidth of $11 \%$. The Q-DPA works with a maximum power gain of 24.16.

Keywords: Doherty, High Efficiency, Load Modulation, PAE, Wideband.

\section{Introduction}

In the era of traveling to untouchable space spots, the nadir of the oceans, wirelessly connected systems are strongly needed. The world of wireless networks is interminably insatiable for developing the current high speed and high-efficiency subsystems for the sake of realizing a modern, power-efficient globe. This future world's perspective entices researchers to introduce modified structures and theories for the communication subsystems. By studying the field of designing PAs, in retrospect, it can be understood several different PA design methods are introduced to increase PAs' PAE, bandwidth, to name but a handful. Such methods comprise envelope tracking [1-5], switching mode class PAs [6-10], harmonically tuned class PAs [10-15], Cherix outphasing [16-20], and DPA [21-25]. DPA is one such PA that is still under more scrutiny to subdue the related restrictions of exerted technology and try to reach the best possible performance of that PA [26-30]. This PA comprises two power amplification paths. The first one is called main; its related PA works as class AB-PA and contributes to amplification in the whole input power range. The second path is named auxiliary and is biased as a class C PA. The latter path is only active when the input level power increases. Hereafter we call this power region the high-power region. To prevent efficiency degradation due to saturation of the main power amplifier in the high-power region, load modulation is done at the output of $\mathrm{PA}_{\text {main }}$ using a $\lambda / 4$

TL. Many developing strategies have been applied to the conventional DPA [25]. The common 
point of almost all of them is that these designs are based on a $\lambda / 4$ TL-based load modulation in which $Z_{\text {out,main }}$ trajectory change in power, and frequency sweep are not precisely modeled. According to [23], revising load modulation can be done based on linear modeling of the variations in $\mathrm{Z}_{\mathrm{out}, \text { main }}$ 's trajectory in power sweep at modulation equation. This load modulation modification led to the PAE increase in comparison by the condition in which a conventional modulation was applied to DPA. Since that revised modulation-based DPA does not provide a broadband performance, in order to design a wideband DPA, more consideration should be attended to. In this work, to present a high PAE and wideband DPA in GaAs technology in which $\mathrm{Z}_{\text {out,main }}$ shows two disparate trajectories in power and frequency sweeps, the strategy of load modulation is modified.

Fulfilling an ideal wideband load modulation that precisely follows $\mathrm{Z}_{\text {out,main }}$ variation in power sweep leads to significant complexity in design. Therefore, in this work, active wideband feedback is applied to $\mathrm{PA}_{\text {main }}$ to reach this $\mathrm{PA}$ to its high efficiency in high power region. $\mathrm{PA}_{\text {main }}$ design is done by keeping it away from saturation before presenting its highest efficiency. According to the non-saturated status of the $\mathrm{PA}_{\text {main }}$, point-to-point narrowband following of $\mathrm{Z}_{\mathrm{out} \text {,main }}$ variation in high power region is not needed anymore. Expunging the narrowband modulation can pave the way to design a wideband DPA.

Furthermore, bias and size selection of $\mathrm{PA}_{\text {feedback }}$ should be made to reach the required Pout feedback while its consumed power is not high. Such a design of feedback path contributes to keeping the PAE as high as possible. Analytical studies of $\mathrm{PA}_{\text {feedback }}$ design for PAE's sake are done in the next section, design theory.

\section{Design Theory}




\section{Active feedback utilization}

According to the conventional DPA concept, in the high-power region, when PAaux contributes to power amplification, $\lambda / 4$ TL-based load modulation averts DPA's efficiency roll-off [25].

However, this approach has its own disadvantages: bandwidth degradation due to narrowband behavior of $\lambda / 4 \mathrm{TL}$, not expecting $Z_{\text {out,main }}$ variation with power in which such changes are needed to be applied in a revised load modulation [23], to name but a handful. Load modulation begins when PAaux commences power amplification. As it can be construed from Eq.1, $i_{\text {aux }} / i_{\text {main }}$ term plays a role in $Z_{\text {out,main }}$ decrease by which DPA's efficiency roll-off can be prevented.

$$
Z_{\text {Mout, main }}=\frac{Z_{0}^{2}}{R_{L}}-Z_{0} \frac{i_{\text {aux }}}{i_{\text {main }}}
$$

Still, it highly depends on $\lambda / 4$ TL usage to fulfill the task of modulation. By considering $\lambda / 4 \mathrm{TL}$ inherent narrowband feature and load modulation lockage in multi-stage DPA[Grebini], some modifications for the load modulation approach in some cases are needed. Class AB PAs are designed for providing the maximum efficiency in the highest input power level, and after that, applying higher Pin leads to their considerable efficiency roll-off. In this work, we designed PA $\mathrm{A}_{\text {main }}$ so that it does not reach its maximum efficiency at the end of the low-power region. Active feedback starts injecting extra power to PAmain's second stage as power increases-whole PA works in the high-power region. By doing so, $\mathrm{PA}_{\text {main }}$ provides its maximum drain efficiency when the input power is high.

\section{Efficiency concerns in the presence of active feedback}


From another scope, to have a higher efficiency of $\mathrm{PA}_{\text {main }}$ and active feedback than conventional AB-class PA in TL-based DPA, more considerations should analytically discuss that hereunder we delved into them.

First, the total efficiency of $\mathrm{PA}_{\text {main }}$ and the active feedback should be more than $\mathrm{PA}_{\text {main }}$ in conventional DPA. To realize this efficiency priority, the consumed power of PAfeedback should follow a restriction by which presenting higher efficiency of the feedback-based PA is guaranteed.

The following equations mathematically show how this condition can be satisfied.

$$
\begin{aligned}
& \frac{\left(0.9 P_{i n, A B}+P_{\text {out }, \text { feedback }}\right) G_{A B}+P_{\text {out }, C}}{\frac{\left(0.9 P_{\text {in }, A B}+P_{\text {out }, \text { feedback }}\right) G_{A B}}{\eta_{A B}}+P_{D C, \text { feedback }}+\frac{P_{\text {out }, C}}{\eta_{C}}} \geq \frac{\left(P_{i n, A B}\right) G_{A B}+P_{\text {out }, C}}{\frac{\left(P_{i n, A B}\right) G_{A B}}{\eta_{A B}}+\frac{P_{\text {out }, C}}{\eta_{C}}} \\
& P_{D C, \text { feedback }} \leq \frac{P_{\text {out }, \text { feedback }} G_{A B}\left(\eta_{C}\left(P_{i n, A B} G_{A B}\right)+\eta_{A B} P_{\text {out }, C}\right)}{\eta_{C} \eta_{A B}\left(P_{i n, A B} G_{A B}+P_{o u t, C}\right)}
\end{aligned}
$$

The restriction of Eq.3 indicates the condition for bias and size selection of $\mathrm{PA}_{\text {feedback, }}$ a $6 \times 150$ transistor with $-1 \mathrm{~V}$ and $5 \mathrm{~V}$ bias voltages for its gate and drain.

\section{Feedback junction to the main path}

One of the typical conjunction of the feedback path to the main loop is the resistive one. Due to power dissipation in this method, capacitive or inductive ones are preferred. In the case of the capacitive junction, due to the degradation effect of large capacitors in the circuit's performance and realization in MMIC, inductive junction is selected.

Among several configurations of the inductive junction, quasi T-junction is chosen. The insertion of this junction should be so that no significant bandwidth degradation has occurred in OMN of the main path and whole DPA. Since OMNs designing both paths are correlated together, bandwidth degradation prevention due to inductive junction insertion should also be done. To deal 
with this issue, both networks are designed with a bandwidth margin to ensure that even with inductive insertion, the proposed DPA will provide the desired bandwidth. Figure 1. exhibits the bandwidth of inductive junction, OMNs of PAmain, and PAaux. This figure proves that the DPA is able to provide the aimed bandwidth.

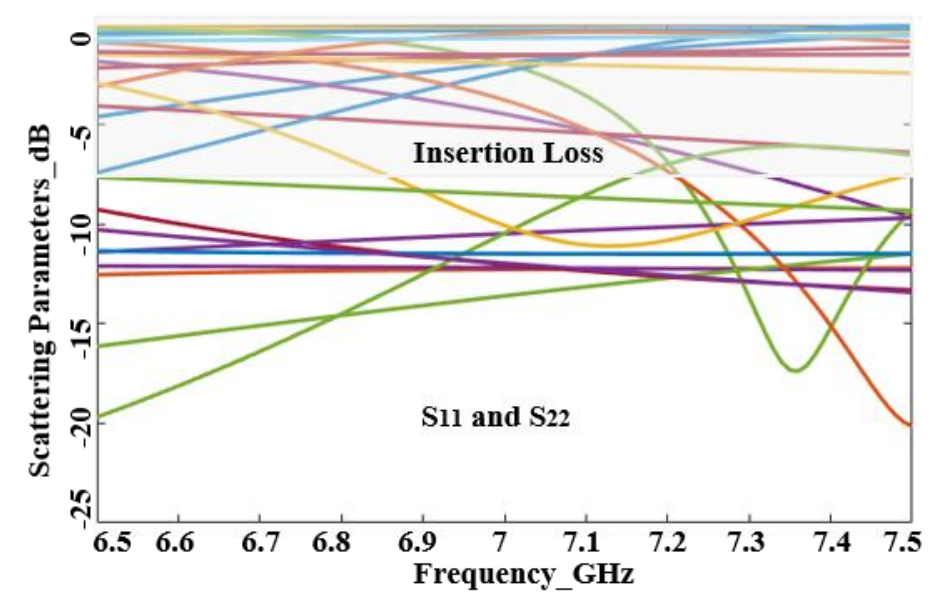

Figure 1. Scattering parameters of the proposed Q-DPA matching networks

\section{Class of PAfeedback selection}

To satisfy Eq.2, designing $\mathrm{PA}_{\text {active }}$ in class $\mathrm{C}$ seems to be a proper strategy to take. Still, by considering class C PA's inherent narrowband performance, some more considerations for choosing the class in which $\mathrm{PA}_{\text {feedback }}$ operates should be scrutinized. For the sake of presenting a wideband performance, $\mathrm{PA}_{\text {feedback }}$ can be biased as an $\mathrm{AB}$-class $\mathrm{PA}$, and for controlling its consumed power, the drain voltage of $\mathrm{PA}_{\text {feedback }}$ should be as low as possible.

In the aspect of stability, two main issues should be addressed here: preventing oscillation after applying active feedback in the circuit. The other is the transistor's stability. For the sake of coping with undesired oscillation due to feedback presence, prevention of the ring oscillator presence, feedback path mandatorily should enjoy the odd number of PA stages so that total there are even

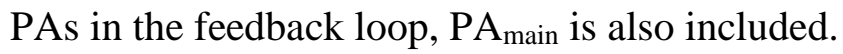


As a consequence, the recurrent signal of the active feedback to $\mathrm{PA}_{\text {main }}$ should undergo 180 phase change, which can obtain by common source figuration. By considering the desired $\mathrm{P}_{\text {out,feedback, }}$ a one-stage common source structure is selected for $\mathrm{PA}_{\text {feedback. }}$ To deal with the transistor's stability as a by default unstable device, large-sized transistor usage in this technology needs to utilizing a low-resistance resistor in the transistors' stabilizer network. Such a network has the most negligible degradation on transistor's gain; therefore, the desired output power can be delivered to the input of $\mathrm{PA}_{\text {main,2nd stage. }}$

For the sake of the non-saturated status of $\mathrm{PA}_{\text {feedback }}$ in the high power level, the capacitance of the capacitor in that PA's stabilizer network is set in a medium value to presents proper power gain while it is not saturated. It should be noticed that properly low-Q LC networks design matching networks of $\mathrm{PA}_{\text {feedback}}$, and this point is valid for all other matching networks for $\mathrm{PA}_{\text {main }}$ and PAauxiliary. These appropriate low-Q networks present the desired bandwidth (see Figure 1.).

\section{Input power divider}

According to the strategy of this work to prevent $\mathrm{PA}_{\text {main }}$ saturation during high-power region, input power should be controlled so that the main application path delivers a small portion of power. According to what proposed in [31], the characteristic impedance of main branch is matched to main path's optimum impedance at low power level to satisfy this condition. The auxiliary branch's impedance characteristic is matched to the optimum impedance of the auxiliary amplification path. By doing so in the high power region, most of the input power is assigned to the auxiliary power amplification path.

\section{Phase compensation}


In the conventional DPA, a $\lambda / 4$ TL at the input of the auxiliary path of amplification is placed to compensate for the $90^{\circ}$ difference in the main path phase. This difference is due to the $\lambda / 4 \mathrm{TL}$ contributing to the conventional load modulation. In this work, by modifying the strategy of modulation, the second $\lambda / 4$ TL utilization is not needed anymore. Still, due to the difference in these two paths of amplification, both paths' consequent phase differences should be compensated. According to [21], the difference in the phase of main and auxiliary paths is compensated in designing the matching networks of main path.

\section{Bandwidth}

One of the primary concern in this design is providing wideband performance. To satisfy the desired $11 \%$ fractional bandwidth with a revised approach for load modulation, all sections of DPA, such as input power divider and matching networks, should provide the wideband performance. Lumped model of the Wilkinson power divider presents the aimed $11 \%$ bandwidth. Besides, the appropriately low-Q LC networks can serve as wideband matching networks. Figure 1. shows the bandwidth of each section and the whole DPA. It can be easily understood that the desired bandwidth is reached.

Power combination concerns

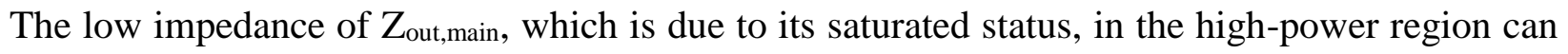
destructively affect the power combination of DPA's output port. According to DPA's concept [25], to address this issue, the $\lambda / 4$ TL-based load modulation plays a role. In our case, in which saturation of $\mathrm{PA}_{\text {main }}$ is prevented, the output impedance of this PA doesn't engender any concern. It should be noticed the only thing worthy of attention is the output matching networks of both 
paths should be designed so that the output port of our proposed Q-DPA be matched to standard $50 \Omega$. Figure 2. shows the output impedance of main and auxiliary paths of amplification. The resultant impedance is matched to the mentioned standard one. Figure x1. It also indicates the bandwidth providing by these networks and confirmed their wideband performance.

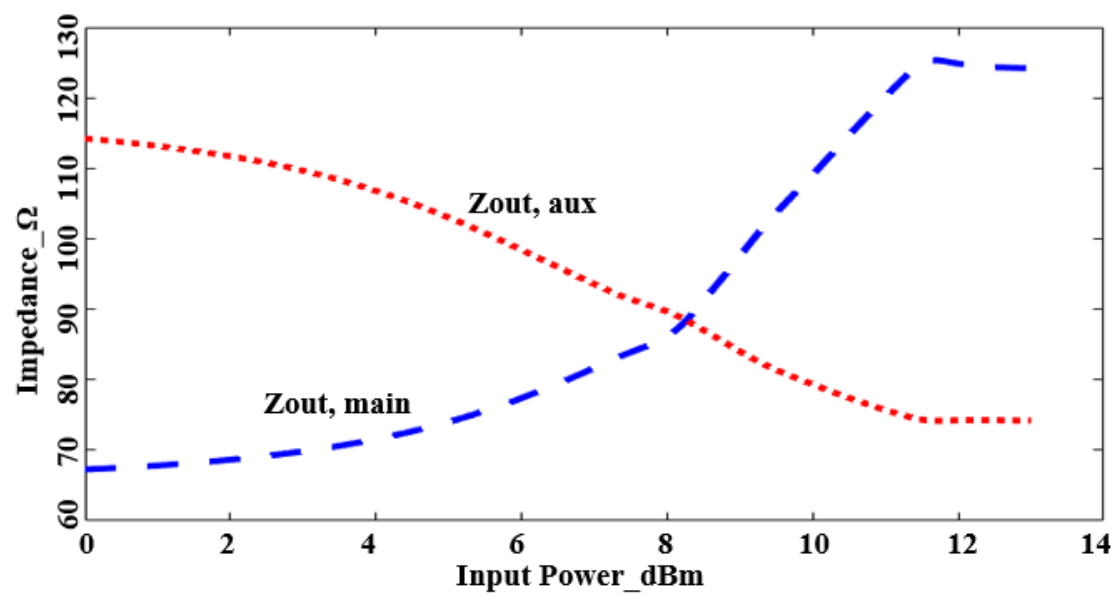

Figure 2. Output impedance of $\mathrm{PA}_{\text {main }}$ and $\mathrm{PA}_{\mathrm{aux}}$

\section{High order harmonic analysis}

Except for harmonic tuned PAs, all other PAs should provide approximately all of their power in the first harmonic for efficiency's sake. In other words, power leakage in other higher harmonics should be averted.

Conventionally, $2^{\text {nd }}$ and $3^{\text {rd }}$ harmonics rejection networks are used in the PA's design. These networks can lead to bandwidth restrictions. A wideband PA can be designed from another scope so that the power level in the $2^{\text {nd }}$ and $3^{\text {rd }}$ harmonics can be neglected. In such a case, for the sake of prevention of the bandwidth degradation, $2^{\text {nd }}$ and $3^{\text {rd }}$ harmonic rejection insertion is skipped. Figures 3. indicate the output power's higher harmonics that are as low as can be ignored. Therefore, the design of harmonic rejection networks is neglected. 


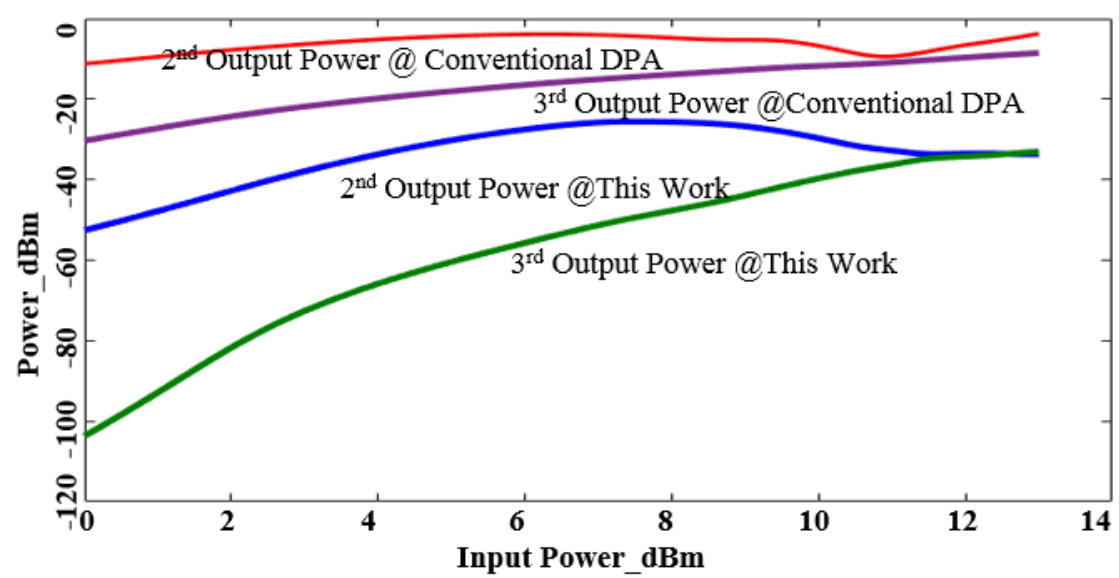

Figure 3. Output power in the second and third harmonics for conventional and the proposed Q-DPA

\section{Comparison}

This work presents a Q-DPA in which active feedback is used. This feedback helps PAmain reaches its maximum efficiency in the high power region. Meanwhile, the input power divider prevents saturation status of $\mathrm{PA}_{\text {main }}$ in low power region. Doing so prevents $\mathrm{PA}_{\text {main }}$ being suffered from nonideal load modulation in which bandwidth degradation and $\mathrm{Z}_{\text {out,main }}$ trajectory in power sweep are not modeled. Therefore, to indicate the proposed Q-DPA's effectiveness, a comparison should be made between feedback-based Q-DPA and conventional one. Figure 4., Figure 5. and Figure 6. show the schematics of conventional and proposed DPAs, respectively. 


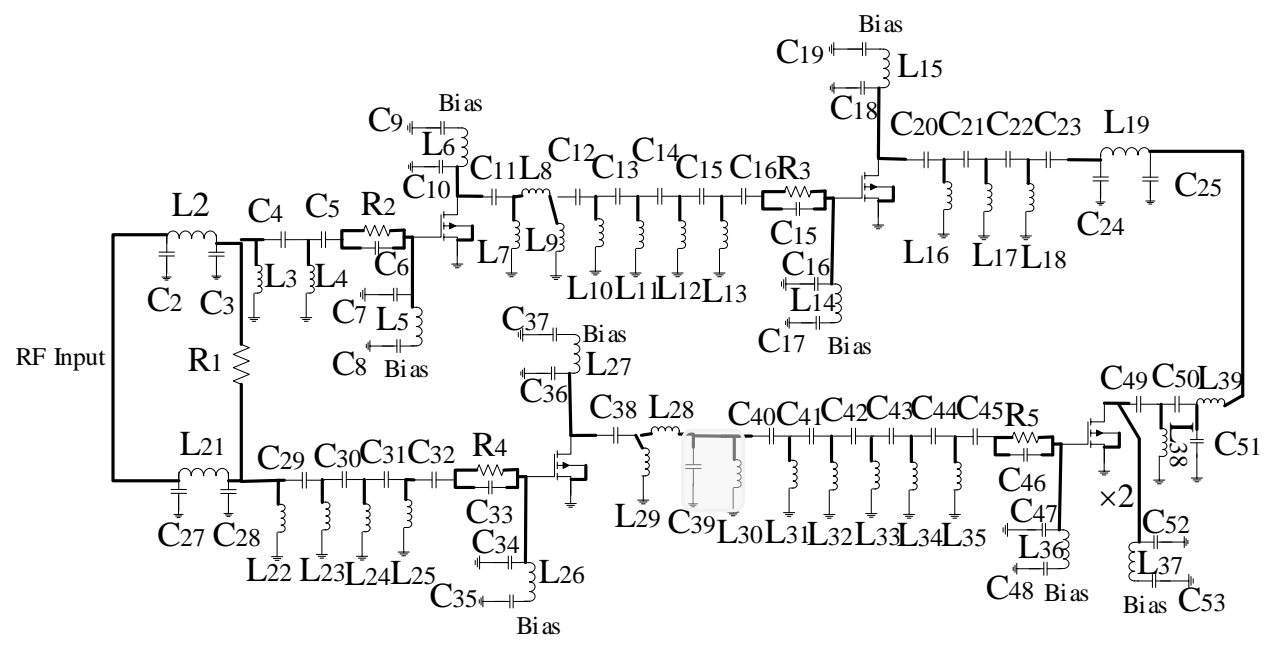

Figure 4. Schematic of the conventional load modulation based DPA (the elements in the grey boxes are the last and the first elements of OMN and IMN of first and second stages PAs, which are merged in circuit's layout, and the exact amount of all elements is provided in Appendix).

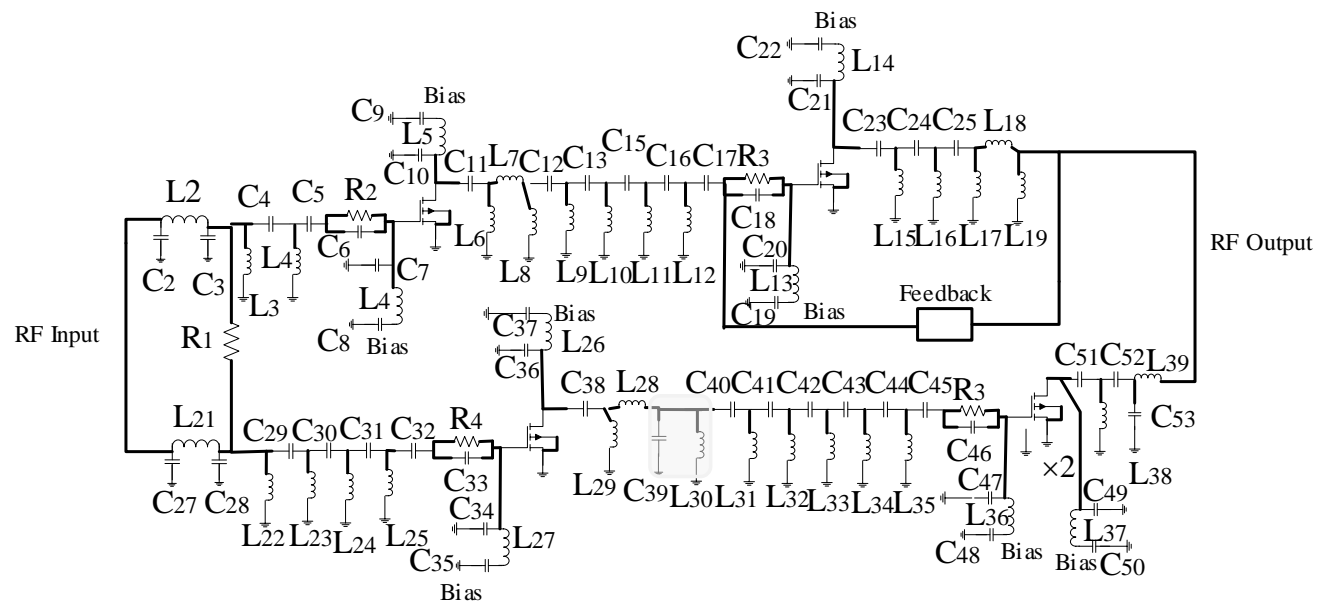

Figure 5. Schematic of the proposed Q-DPA (the elements in grey boxes are the last and the first elements of OMN and IMN of first and second stages PAs, which are merged in circuit's layout, and the exact amount of all elements is provided in Appendix).

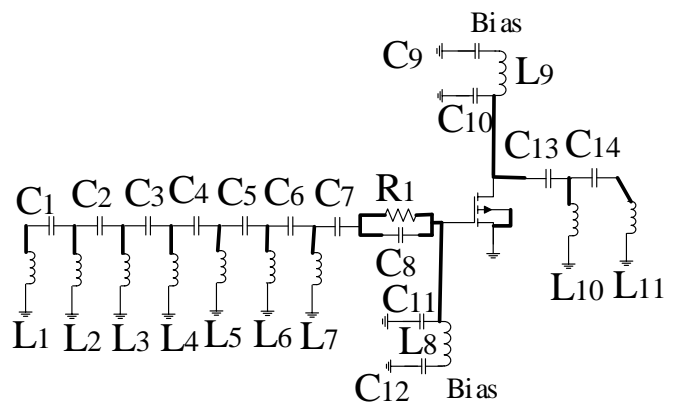

Figure 6. Schematic of the feedback path in the proposed Q-DPA (and the exact amount of all elements is provided in Appendix). 
Figure 7. and Figure 8. depicts PAE, Pout, power gain, and bandwidth of both circuits. It can be construed that utilizing the active feedback leads to $\mathrm{PA}_{\text {main's }}$ 's reaching its maximum efficiency in the high power region.

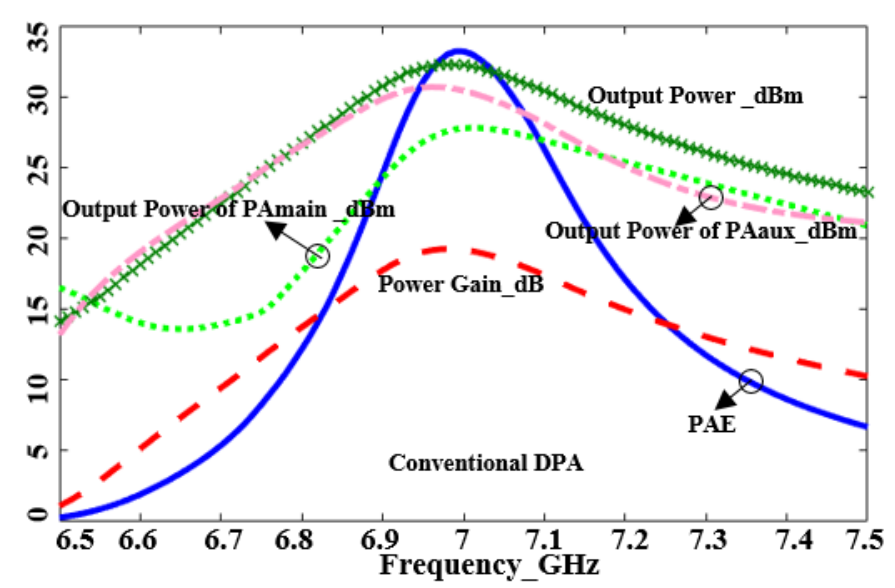

Figure 7. PAE, Power Gain, Output Powers of the conventional DPA

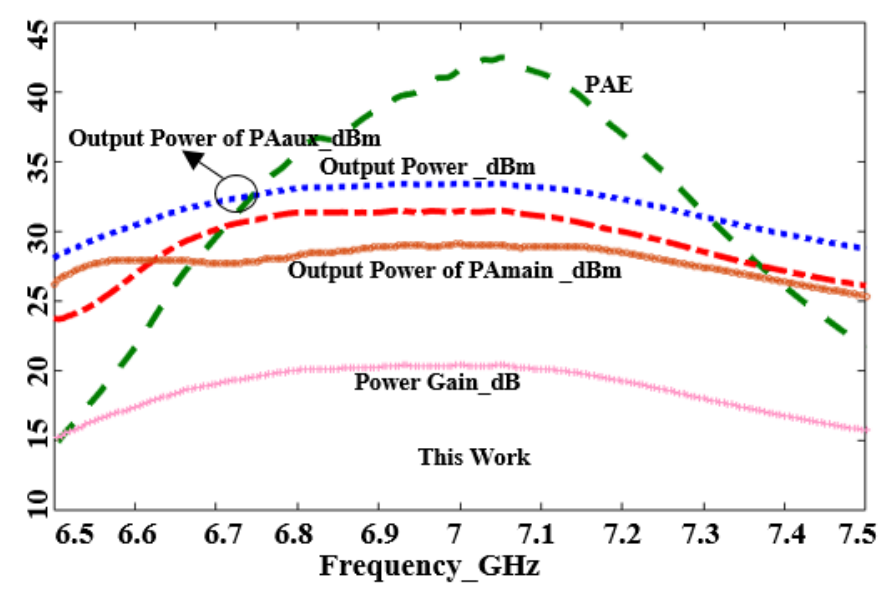

Figure 8. PAE, Power Gain, Output Powers of the proposed Q-DPA

Consequently, utilization of this feedback results in PAE and Pout increase. Furthermore, since $\mathrm{PA}_{\text {main }}$ doesn't saturate during high power region, there is no need to make an ideal load 
modulation by which the exact $\mathrm{Z}_{\mathrm{out} \text {,main }}$ trajectory in power sweep should be followed in order to have the maximum possible PAE.

For a better understand, the performances of both designs in power sweep are shown in Figure 9. and Figure 10. These figures clearly confirm that using the revised approach helps in presenting a high PAE power amplifier.

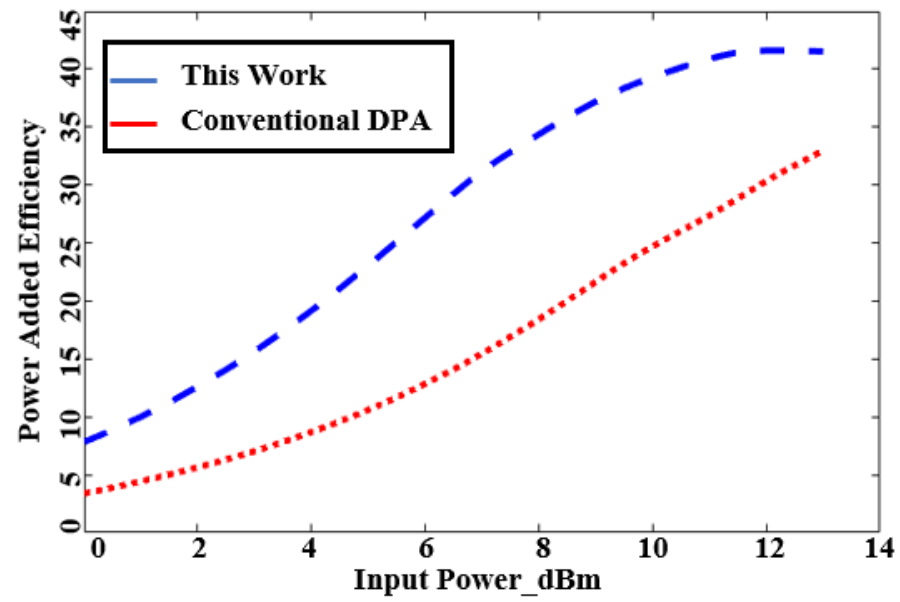

Figure 9. PAE of the conventional and proposed Q-DPA in power sweep

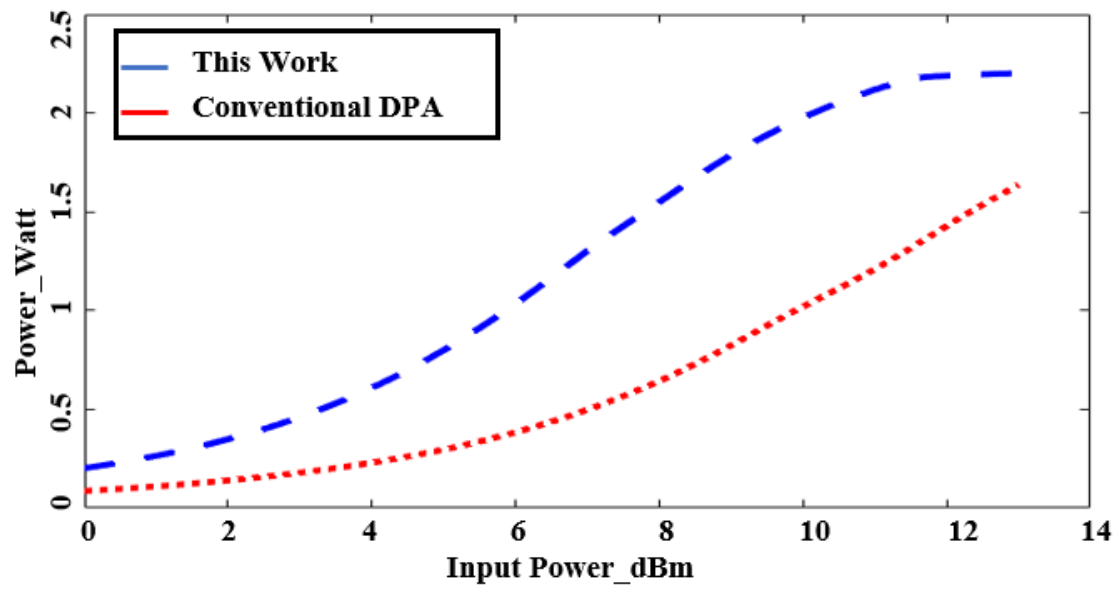

Figure 10. Output Powers of the conventional and proposed Q-DPA in power sweep

To indicate more about the performance of the Figure 11. and Figure 12 show output powers of PAmain, $\mathrm{PA}_{\mathrm{aux}}$, the proposed Q-DPA and its related power gain. 


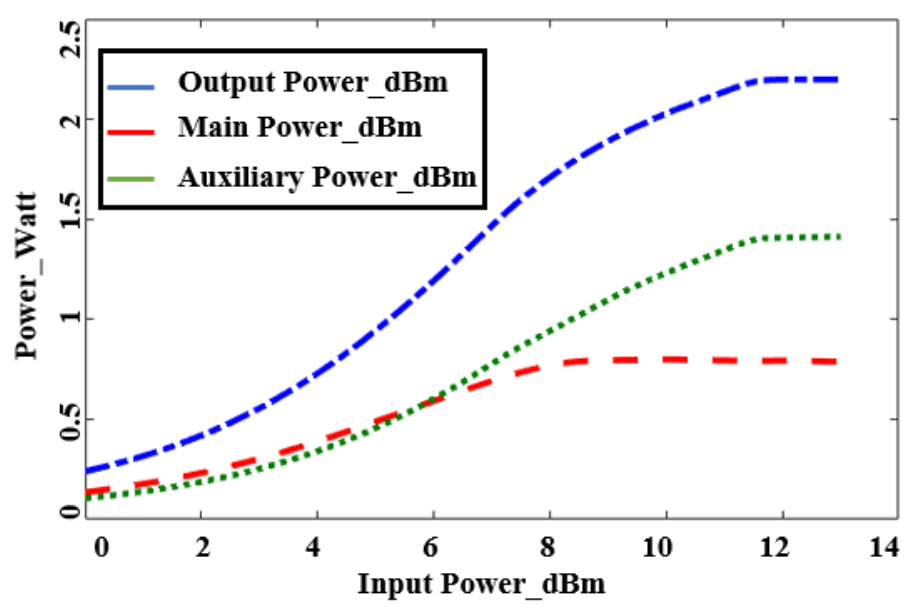

Figure 11. Output Powers of the PAmain, $\mathrm{PA}_{\mathrm{aux}}$, and the proposed Q-DPA in power sweep

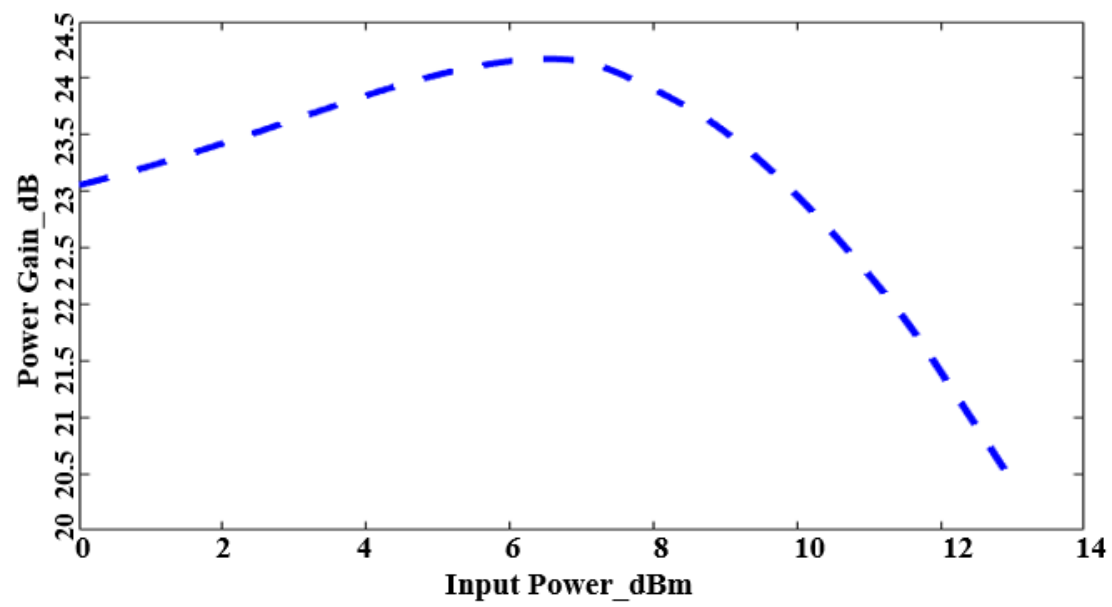

Figure 12. Power Gain of the proposed Q-DPA in power sweep

Table 1. presents the performance comparison of the conventional DPA and the proposed Q-DPA.

This table clearly leads to drawing this conclusion that utilization of the proposed approach in this technology results in PAE and bandwidth development.

Table 1. Comparison of the proposed Q-DPA and Conventional DPA performances

\begin{tabular}{|c|c|c|c|c|c|c|}
\hline & $P_{\text {out }}(\mathrm{dBm})$ & $P_{\text {out } @ 2^{\text {nd }}}(\mathrm{dBm})$ & $P_{\text {out } @ 3^{\text {rd }}}(\mathrm{dBm})$ & Gain $(\mathrm{dB})$ & PAE (\%) & FBW (\%) \\
\hline This Work & 33.4 & -52.5 & -103.6 & 24.16 & 41.59 & 11 \\
\hline $\begin{array}{c}\text { Conventional } \\
\text { DPA }\end{array}$ & 32.3 & -17 & -35.1 & 20.16 & 33.17 & 3.2 \\
\hline
\end{tabular}




\section{Results}

This paper presents a conventional DPA design and an active feedback-based quasi-DPA in GaAs $0,25 \mu \mathrm{m}$ pHEMT MMIC technology. The comparison between these designs depicts the practicality of the proposed Q-DPA. The proposed PA is designed in a two-stage structure for both the main and auxiliary path of amplification. In the main path, at the first stage, $8 \times 150 \mu \mathrm{m}$ transistor is used and has $-1 \mathrm{~V}, 7 \mathrm{~V}$ for its gate and drain bias, respectively. The second one enjoys a single $8 \times 150 \mu \mathrm{m}$ transistor that is biased in $-1 \mathrm{~V}$ and $7.4 \mathrm{~V}$ for its gate and drain. The feedback path comprises a $6 \times 150 \mu \mathrm{m}$ transistor with $-1 \mathrm{~V}$ and $5 \mathrm{~V}$ bias voltages for its gate and drain, respectively. The first stage in the auxiliary path enjoys two parallel $8 \times 150 \mu \mathrm{m}$ transistors that are biased in $-1.2 \mathrm{~V}$ and $7 \mathrm{~V}$ for its gate and drain biasing, respectively, while the second one contains two parallel $8 \times 150 \mu \mathrm{m}$ transistors with $-1.05 \mathrm{~V}$ and $7 \mathrm{~V}$ bias voltages for its gate and drain, respectively. The proposed Q-DPA has the maximum amount for Pout of $33.4 \mathrm{dBm}$, PAE of 41.6, fractional bandwidth of $\mathrm{X}$, which is the frequency range of 6.6-7.4 GHz, the power gain of 24.16 dB. The feedback path's consumed power is designed so that the theory limit (Eq.3) is satisfied.

Table 2. provides the performance comparison among this work and the state of the art DPA designed in GaAs technology, and the Figures 13. Shows the proposed Q-DPA's layout.

Table 2- Performance comparison among the proposed DPA and state of the art DPAs in GaAs technology.

\begin{tabular}{|c|c|c|c|c|c|}
\hline & $P_{\text {out }}(\mathrm{dBm})$ & Gain $(\mathrm{dB})$ & Freq $(\mathrm{GHz})$ & PAE (\%) & Technology \\
\hline$[32]$ & 19.4 & N/A & $62-68$ & 28.3 & $\begin{array}{c}\text { CMOS } \\
0.045 \mu \mathrm{m}\end{array}$ \\
\hline$[33]^{*}$ & $<27$ & $\approx 14$ & 28 & $\approx 37$ & $\begin{array}{c}\text { CMOS } \\
0.055 \mu \mathrm{m}\end{array}$ \\
\cline { 2 - 6 } & $<27$ & $\approx 12$ & 45 & $\approx 35$ & $\begin{array}{c}\text { GaAs } \\
{[34]^{* *}}\end{array}$ \\
\hline $\begin{array}{c}\text { This } \\
\text { Work }\end{array}$ & 27.2 & $<24$ & $5.4-6.2$ & 24.5 & $\begin{array}{c}\text { GaAs } \\
\text { pHEM }\end{array}$ \\
\hline
\end{tabular}




\footnotetext{
* This is a dual-band DPA, and for each band, we reported the best amount of simulation results. The exact amount of most of the criteria is not reported. Therefore, for the code of ethics, each of them has a $\approx$ sign.

** These are the measured results. Simulation results were not reported.
}

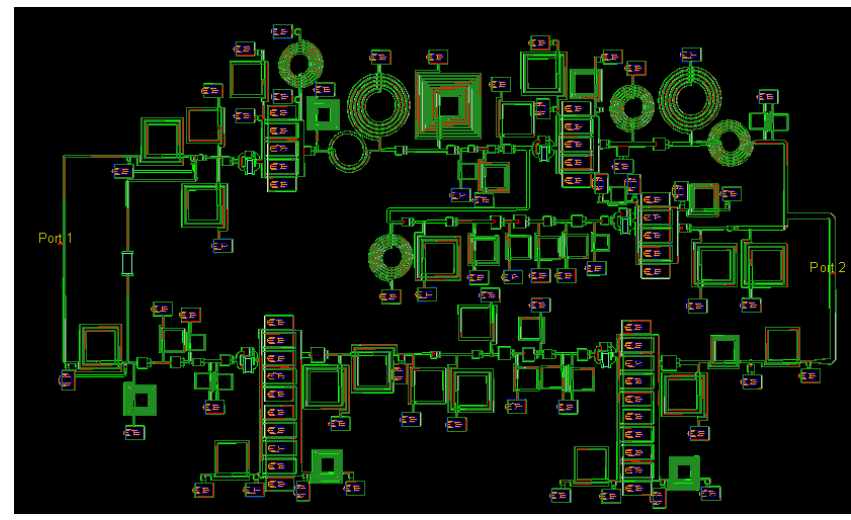

Figure 13. Layout of the proposed Q-DPA

\section{Conclusion}

This work presented the design of a wideband Q-DPA. Considering $\mathrm{Z}_{\text {out,main, }}$ the main variation in power sweep, which makes realizing a proper load modulation hard, is done in the load modulation's revised strategy. That modification is grounded on active feedback. The feedback made $\mathrm{PA}_{\text {main }}$ to be reached its maximum PAE in the highest level of power. Therefore there is no concern about making a delicately ideal load modulation by which increased PAE can be obtained. To draw an accurate conclusion, a comparison between the proposed DPA and that of the conventional DPA in which a $\lambda / 4$ TL-based load modulation is done is made in this work. Both PAs are designed in GaAs pHEMT 0,25 $\mu \mathrm{m}$ MMIC technology in which $\mathrm{Z}_{\text {out,main shows a }}$ considerable variation in power sweep. Besides, $\mathrm{Z}_{\mathrm{out} \text {,main's }}$ trajectories in power and frequency sweeps are not similar. That's why an accurate load modulation considering the trajectory of $\mathrm{Z}_{\mathrm{out}, \mathrm{main}}$ in power sweep cannot be pragmatic for that of $\mathrm{Z}_{\mathrm{out} \text {,main }}$ in frequency change. Therefore, no wideband DPA can be expected. Pout, PAE, the bandwidth of those above mentioned PAs verify that modification in load modulation approach in case of different trajectories of $Z_{\text {out,main }}$ in 
power, and frequency sweeps for a high PAE wideband performance is needed. Because the active feedback in our work consumes power, the dissipated power of that device should be controlled. In this work, this task is done analytically. Besides, $\mathrm{PA}_{\text {feedback's }}$ bias and size selection are also

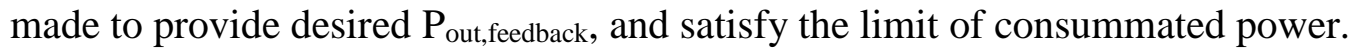

\section{Appendix}

Amount of the elements of the conventional load modulation based DPA (Figure 4):

- $\quad L_{2}=2 \mathrm{nH}, L_{3}=1.85 \mathrm{nH}, L_{4}=0.76 \mathrm{nH}, L_{5}=0.7 \mathrm{nH}, L_{6}=3.2 \mathrm{nH}, L_{7}=3 \mathrm{nH}, L_{8}=0.88 \mathrm{nH}, L_{9}=5.4 \mathrm{nH}, L_{10}=1.6 \mathrm{nH}, L_{11}=0.5$ $\mathrm{nH}, L_{12}=1 \mathrm{nH}, L_{13}=0.72 \mathrm{nH}, L_{14}=1.8 \mathrm{nH}, L_{15}=1.2 \mathrm{nH}, L_{16}=0.78 \mathrm{nH}, L_{17}=1.1 \mathrm{nH}, L_{18}=0.45 \mathrm{nH}, L_{19}=1.4 \mathrm{nH}, \mathrm{nH}$, $L_{21}=2.6 \mathrm{nH}, L_{22}=0.68 \mathrm{nH}, L_{23}=0.36 \mathrm{nH}, L_{24}=0.28 \mathrm{nH}, L_{25}=1.65 \mathrm{nH}, L_{26}=1.77 \mathrm{nH}, L_{29}=1 \mathrm{nH}, L_{30}=1.5 \mathrm{nH}, L_{31}=0.82$ $\mathrm{nH}, L_{32}=0.5 \mathrm{nH}, L_{33}=0.43 \mathrm{nH}, L_{34}=0.25 \mathrm{nH}, L_{35}=0.43 \mathrm{nH}, L_{36}=0.25 \mathrm{nH}, L_{37}=1.85 \mathrm{nH}, L_{38}=1.12 \mathrm{nH}, L_{39}=0.7 \mathrm{nH}$.

- $\quad C_{2}=0.2 \mathrm{pF}, C_{3}=0.2 \mathrm{pF}, C_{4}=0.46 \mathrm{pF}, C_{5}=0.93 \mathrm{pF}, C_{6}=0.3 \mathrm{pF}, C_{7}=0.3 \mathrm{pF}, C_{8}=0.55 \mathrm{pF}, C_{9}=0.3 \mathrm{pF}, C_{10}=0.3 \mathrm{pF}$, $C_{11}=1 \mathrm{pF}, C_{12}=0.3 \mathrm{pF}, C_{13}=0.3 \mathrm{pF}, C_{14}=0.93 \mathrm{pF}, C_{15}=1 \mathrm{pF}, C_{16}=0.58 \mathrm{pF}, C_{17}=0.83 \mathrm{pF}, C_{18}=0.95 \mathrm{pF}, C_{19}=0.3 \mathrm{pF}$, $C_{20}=0.3 \mathrm{pF}, C_{21}=0.7 \mathrm{pF}, C_{22}=1.9 \mathrm{pF}, C_{23}=2.2 \mathrm{pF}, C_{24}=0.2 \mathrm{pF}, C_{25}=0.2 \mathrm{pF}, C_{27}=1.45 \mathrm{pF}, C_{28}=1.8 \mathrm{pF}, C_{29}=0.84$ $\mathrm{pF}, C_{30}=1.4 \mathrm{pF}, C_{31}=0.3 \mathrm{pF}, C_{32}=0.3 \mathrm{pF}, C_{33}=2 \mathrm{pF}, C_{34}=0.3 \mathrm{pF}, C_{35}=0.3 \mathrm{pF}, C_{36}=0.55 \mathrm{pF}, C_{37}=0.5 \mathrm{pF}, C_{38}=0.73$ $\mathrm{pF}, C_{39}=2 \mathrm{pF}, C_{40}=2.26 \mathrm{pF}, C_{41}=1 \mathrm{pF}, C_{42}=1.5 \mathrm{pF}, C_{43}=0.3 \mathrm{pF}, C_{44}=0.3 \mathrm{pF}, C_{45}=2.5 \mathrm{pF}, C_{46}=0.3 \mathrm{pF}, C_{47}=0.3 \mathrm{pF}$, $C_{48}=3 \mathrm{pF}, C_{49}=0.6 \mathrm{pF}, C_{50}=0.18 \mathrm{pF}$.

- $\quad R_{1}=100 \Omega, R_{2}=20 \Omega, R_{3}=20 \Omega, R_{4}=20 \Omega, R_{5}=20 \Omega$.

Amount of the elements of the Proposed DPA (Figure 5):

- $\quad L_{2}=2 \mathrm{nH}, L_{3}=1.85 \mathrm{nH}, L_{4}=0.76 \mathrm{nH}, L_{5}=0.7 \mathrm{nH}, L_{6}=3.2 \mathrm{nH}, L_{7}=3 \mathrm{nH}, L_{8}=0.88 \mathrm{nH}, L_{9}=5.4 \mathrm{nH}, L_{10}=1.6 \mathrm{nH}, L_{11}=0.5$ $\mathrm{nH}, L_{12}=1 \mathrm{nH}, L_{13}=0.72 \mathrm{nH}, L_{14}=1.8 \mathrm{nH}, L_{15}=1.2 \mathrm{nH}, L_{16}=4 \mathrm{nH}, L_{17}=6 \mathrm{nH}, L_{18}=5 \mathrm{nH}, L_{19}=3.3 \mathrm{nH}, L_{21}=0.2 \mathrm{nH}$, $L_{22}=1.98 \mathrm{nH}, L_{23}=2.6 \mathrm{nH}, L_{24}=0.68 \mathrm{nH}, L_{25}=0.36 \mathrm{nH}, L_{26}=0.28 \mathrm{nH}, L_{27}=0.65 \mathrm{nH}, L_{28}=3.5 \mathrm{nH}, L_{29}=1.65 \mathrm{nH}$, $L_{30}=1.97 \mathrm{nH}, L_{31}=0.75 \mathrm{nH}, L_{32}=1.5 \mathrm{nH}, L_{33}=0.82 \mathrm{nH}, L_{34}=0.53 \mathrm{nH}, L_{35}=0.43 \mathrm{nH}, L_{36}=0.25 \mathrm{nH}, L_{37}=0.9 \mathrm{nH}, L_{38}=4$ $\mathrm{nH}, L_{39}=1.85 \mathrm{nH}, L_{40}=1.12 \mathrm{nH}, L_{41}=0.7 \mathrm{nH}$.

- $\quad C_{2}=0.2 \mathrm{pF}, C_{3}=0.2 \mathrm{pF}, C_{4}=0.46 \mathrm{pF}, C_{5}=0.93 \mathrm{pF}, C_{6}=0.3 \mathrm{pF}, C_{7}=0.3 \mathrm{pF}, C_{8}=0.55 \mathrm{pF}, C_{9}=0.3 \mathrm{pF}, C_{10}=0.3 \mathrm{pF}$, $C_{11}=1 \mathrm{pF}, C_{12}=0.3 \mathrm{pF}, C_{13}=0.3 \mathrm{pF}, C_{14}=0.93 \mathrm{pF}, C_{15}=1 \mathrm{pF}, C_{16}=0.58 \mathrm{pF}, C_{17}=0.83 \mathrm{pF}, C_{18}=0.95 \mathrm{pF}, C_{19}=0.3 \mathrm{pF}$, $C_{20}=0.3 \mathrm{pF}, C_{21}=0.7 \mathrm{pF}, C_{22}=1.9 \mathrm{pF}, C_{23}=2.2 \mathrm{pF}, C_{24}=0.2 \mathrm{pF}, C_{25}=0.2 \mathrm{pF}, C_{27}=1.45 \mathrm{pF}, C_{28}=1.8 \mathrm{pF}, C_{29}=0.84$ $\mathrm{pF}, C_{30}=1.4 \mathrm{pF}, C_{31}=0.3 \mathrm{pF}, C_{32}=0.3 \mathrm{pF}, C_{33}=2.9 \mathrm{pF}, C_{34}=0.3 \mathrm{pF}, C_{35}=0.3 \mathrm{pF}, C_{36}=0.55 \mathrm{pF}, C_{37}=0.5 \mathrm{pF}, C_{38}=0.73$ 
$\mathrm{pF}, C_{39}=2 \mathrm{pF}, C_{40}=2.26 \mathrm{pF}, C_{41}=1 \mathrm{pF}, C_{42}=1.5 \mathrm{pF}, C_{43}=0.3 \mathrm{pF}, C_{44}=0.3 \mathrm{pF}, C_{45}=3.5 \mathrm{pF}, C_{46}=0.3 \mathrm{pF}, C_{47}=0.3 \mathrm{pF}$,

$C_{48}=3 \mathrm{pF}, C_{49}=0.6 \mathrm{pF}, C_{50}=0.18 \mathrm{pF}$.

- $\quad R_{1}=100 \Omega, R_{2}=20 \Omega, R_{3}=20 \Omega, R_{4}=20 \Omega, R_{5}=20 \Omega$.

Amount of the elements of the Feedback path (Figure 6):

- $\quad L_{1}=3.6 \mathrm{nH}, L_{2}=2.6 \mathrm{nH}, L_{3}=1.3 \mathrm{nH}, L_{4}=0.64 \mathrm{nH}, L_{5}=0.64 \mathrm{nH}, L_{6}=0.5 \mathrm{nH}, L_{7}=0.47 \mathrm{nH}, L_{8}=1 \mathrm{nH}, L_{9}=0.7 \mathrm{nH}$,

$L_{10}=2.26 \mathrm{nH}, L_{11}=2.45 \mathrm{nH}$

- $\quad C_{1}=1.5 \mathrm{pF}, C_{2}=0.86 \mathrm{pF}, C_{3}=1.8 \mathrm{pF}, C_{4}=2.45 \mathrm{pF}, C_{5}=1.3 \mathrm{pF}, C_{6}=1.88 \mathrm{pF}, C_{7}=0.81 \mathrm{pF}, C_{8}=0.85 \mathrm{pF}, C_{9}=0.28 \mathrm{pF}$,

$C_{10}=0.28 \mathrm{pF}, C_{11}=0.28 \mathrm{pF}, C_{12}=0.28 \mathrm{pF} C_{13}=0.92 \mathrm{pF}, C_{14}=0.24 \mathrm{pF}, C_{15}=0.58 \mathrm{pF}$

- $\quad R_{3}=20 \Omega$

\section{References}

[1] Kwan, Andrew K., et al. "Linearization of a highly nonlinear envelope tracking power amplifier targeting maximum efficiency." IEEE Microwave and Wireless Components Letters 27.1 (2016): 82-84.

[2] Jeong, Junhyung, Girdhari Chaudhary, and Yongchae Jeong. "Time mismatch effect in linearity of hybrid envelope tracking power amplifier." IEEE Microwave and Wireless Components Letters 25.8 (2015): 550552.

[3] Kim, Woo-Young, et al. "A CMOS envelope-tracking transmitter with an on-chip common-gate voltage modulation linearizer." IEEE microwave and wireless components letters 24.6 (2014): 406-408.

[4] Sarbishaei, Hassan, et al. "Dual-band Volterra series digital pre-distortion for envelope tracking power amplifiers." IEEE Microwave and wireless Components letters 24.6 (2014): 430-432.

[5] Moon, Kyunghoon, et al. "An HBT saturated power amplifier with minimized knee effect for envelope tracking operation." IEEE Microwave and Wireless Components Letters 25.8 (2015): 544-546.

[6] Ozen, Mustafa, Rik Jos, and Christian Fager. "Continuous class-E power amplifier modes." IEEE Transactions on Circuits and Systems II: Express Briefs 59.11 (2012): 731-735.

[7] Albulet, Mihai, and Robert E. Zulinski. "Effect of switch duty ratio on the performance of class E amplifiers and frequency multipliers." IEEE Transactions on Circuits and Systems I: Fundamental Theory and Applications 45.4 (1998): 325-335.

[8] Aflaki, Pouya, Renato Negra, and Fadhel M. Ghannouchi. "Dedicated large-signal GaN HEMT model for switching-mode circuit analysis and design." IEEE Microwave and Wireless Components Letters 19.11 (2009): 740-742.

[9] Telegdy, Attila, Bela Molnar, and Nathan O. Sokal. "Class-E/sub M/switching-mode tuned power amplifierhigh efficiency with slow-switching transistor." IEEE transactions on microwave theory and techniques 51.6 (2003): 1662-1676.

[10] Kitchen, Jennifer N., et al. "Polar SiGe class $\mathrm{E}$ and $\mathrm{F}$ amplifiers using switch-mode supply modulation." IEEE Transactions on Microwave Theory and Techniques 55.5 (2007): 845-856.

[11] Sharma, Tushar, et al. "Generalized continuous class-F harmonic tuned power amplifiers." IEEE Microwave and Wireless Components Letters 26.3 (2016): 213-215. 
[12] Nawaz, Asad A., et al. "Harmonic tuning of stacked sige power amplifiers using active load pull." IEEE Microwave and Wireless Components Letters 28.3 (2018): 245-247.

[13] Xia, Jing, Xiao-Wei Zhu, and Lei Zhang. "A linearized 2-3.5 GHz highly efficient harmonic-tuned power amplifier exploiting stepped-impedance filtering matching network." IEEE Microwave and Wireless Components Letters 24.9 (2014): 602-604.

[14] Cheng, Qian-Fu, et al. "Two-stage high-efficiency concurrent dual-band harmonic-tuned power amplifier." IEEE Transactions on Microwave Theory and Techniques 64.10 (2016): 3232-3243.

[15] Merrick, Brian M., Justin B. King, and Thomas J. Brazil. "The continuous harmonic-tuned power amplifier." IEEE Microwave and Wireless Components Letters 25.11 (2015): 736-738.

[16] Banerjee, Aritra, Lei Ding, and Rahmi Hezar. "A High Efficiency Multi-Mode Outphasing RF Power Amplifier With $31.6 \mathrm{dBm}$ Peak Output Power in 45nm CMOS." IEEE Transactions on Circuits and Systems I: Regular Papers 67.3 (2020): 815-828.

[17] Hung, Tsai-Pi, et al. "CMOS outphasing class-D amplifier with Chireix combiner." IEEE Microwave and Wireless Components Letters 17.8 (2007): 619-621.

[18] Chen, Jau-Horng. "An efficiency-improved outphasing power amplifier using RF pulse modulation." IEEE microwave and wireless components letters 20.12 (2010): 684-686.

[19] Ning, Kang, et al. "A 30-GHz CMOS SOI outphasing power amplifier with current mode combining for high backoff efficiency and constant envelope operation." IEEE Journal of Solid-State Circuits 55.5 (2019): 14111421.

[20] Barton, Taylor W., and David J. Perreault. "Theory and implementation of RF-input outphasing power amplification." IEEE Transactions on Microwave Theory and Techniques 63.12 (2015): 4273-4283.

[21] Rouhani, Seyedehmarzieh, et al. "A wideband quasi-asymmetric Doherty power amplifier with a two-section matching-phase difference compensator network design using GaAs technology." Analog Integrated Circuits and Signal Processing 105.3 (2020): 359-370.

[22] Jia, Pei, Fei You, and Songbai He. "A 1.8-3.4-GHz bandwidth-improved reconfigurable mode Doherty power amplifier utilizing switches." IEEE Microwave and Wireless Components Letters 30.1 (2019): 102105 .

[23] Rouhani, Seyedehmarzieh, et al. "Increased Efficiency of a Current Compensating Load Modulation Based MMIC Doherty Power Amplifier Design In 0.25 m GaAs pHEMT Technology." ScienceOpen Preprints (2020).

[24] Chen, Yen-Chih, et al. "A Ka-band transformer-based Doherty power amplifier for multi-Gb/s application in 90-nm CMOS." IEEE Microwave and Wireless Components Letters 28.12 (2018): 1134-1136.

[25] Doherty, William H. "A new high efficiency power amplifier for modulated waves." Proceedings of the Institute of Radio Engineers 24.9 (1936): 1163-1182.

[26] Cao, Yuchen, Haifeng Lyu, and Kenle Chen. "Asymmetrical Load Modulated Balanced Amplifier With Continuum of Modulation Ratio and Dual-Octave Bandwidth." IEEE Transactions on Microwave Theory and Techniques 69.1 (2020): 682-696.

[27] Srirattana, Nuttapong, et al. "Analysis and design of a high-efficiency multistage Doherty power amplifier for wireless communications." IEEE Transactions on Microwave Theory and Techniques 53.3 (2005): 852860 . 
[28] Hasin, Muhammad Ruhul, and Jennifer Kitchen. "Optimized load trajectory for finite peaking off-state impedance-based Doherty power amplifiers." IEEE Microwave and Wireless Components Letters 29.7 (2019): 486-488.

[29] Kim, Joonhyung. "Highly efficient asymmetric class-F-1/F GaN Doherty amplifier." IEEE Transactions on Microwave Theory and Techniques 66.9 (2018): 4070-4077.

[30] Kim, Joon Hyung, and Chul Soon Park. "Analysis and implementation of Doherty power amplifier with twopoint envelope modulation." IEEE transactions on microwave theory and techniques 60.5 (2012): 13531364.

[31] Kang, Daehyun, et al. "Design of bandwidth-enhanced Doherty power amplifiers for handset applications." IEEE Transactions on Microwave Theory and Techniques 59.12 (2011): 3474-3483

[32] Nguyen, Huy Thong, et al. "A linear high-efficiency millimeter-wave CMOS Doherty radiator leveraging multi-feed on-antenna active load modulation." IEEE Journal of Solid-State Circuits 53.12 (2018): 35873598.

[33] Lv, Guansheng, et al. "A Compact Ka/Q Dual-Band GaAs MMIC Doherty Power Amplifier With Simplified Offset Lines for 5G Applications." IEEE Transactions on Microwave Theory and Techniques 67.7 (2019): 3110-3121.

[34] Jung, Doohwan, Huan Zhao, and Hua Wang. "A CMOS highly linear Doherty power amplifier with multigated transistors." IEEE Transactions on Microwave Theory and Techniques 67.5 (2019): 1883-1891. 